\title{
ELASTICIDADE PREÇO E RENDA DA DEMANDA POR ENERGIA ELÉTRICA NAS REGIÕES BRASILEIRAS: UMA ABORDAGEM ATRAVÉS DE PAINEL DINÂMICO
}

\author{
Fabiano da Costa Dantas* \\ Edward Martins Costa * \\ J orge Luiz Mariano da Silva
}

\begin{abstract}
Resumo
Esse trabalho tem como objetivo estimar as elasticidades preço e renda da demanda residencial, industrial e comercial de energia elétrica das regiões brasileiras, durante o período de 2000 a 2015. Partindo da hipótese de que o avanço tecnológico, o crescimento econômico e a melhoria da qualidade de vida no país implicaram em mudanças nas elasticidades preço e rendas da demanda por energia elétrica. Para tal, utilizou-se a metodologia de dados em painel, estimado pelo Método dos Momentos Generalizados (GMM), em uma versão conhecida como System-GMM. Os resultados mostraram que a elasticidaderenda da classe de consumo industrial foi a que apresentou os maiores valores em todas as regiões analisadas, se comparado com as outras classes estudadas. Constatou-se ainda quea classe comercial foi a que registrou os maiores impactos na elasticidade-preço em todas as regiões brasileiras, o que não divergem muito das análises ocorridas por outros estudos, entretanto com outras metodologias.
\end{abstract}

Palavras-Chave: Elasticidade. Energia Elétrica. Dados em Painel.

\section{Abstract}

This study aims to estimate the price and income elasticities of residential demand, industrial and commercial power of the Brazilian regions during the period 2000 to 2015. Assuming that technological advancement, economic growth and improving the quality of life in the country imply changes in price and income elasticities of demand for electricity. To this end, we used the methodology of panel data, estimated by the Generalized Method of Moments (GMM) in a version known as System-GMM. The results showed that the income elasticity of the class of industrial consumption showed the highest values in all regions examined, compared with the other classes studied. It was further observed that the commercial class was the one that recorded the greatest impact on the price elasticity in all Brazilian regions, which do not differ much from the analyzes that have occurred in other studies, however with other methodologies.

Keywords: Elasticity. Electric Power. Panel data.

J EL: C33, D12, L94.

* Universidade Federal Rural do Semiárido - UFERSA

- Universidade Federal do Ceará - UFC

^ Universidade Federal do Rio Grande do Norte - UFRN 


\section{Introdução}

A estabilidade econômica alcançada após a implantação do Plano Real em 1994 levou a economia nacional a retomada do crescimento econômico, através do controle da inflação, do aumento da renda da população, etambém pelo aumento na oferta por energia elétrica. As taxas de expansão da oferta no setor elétrico até a primeira metade da década de 90 apresentaram elevados índices, fundamentados principalmente nas disponibilidades de autofinanciamento por meio de tarifas reais, pelos recursos oriundos do Governo Federal e o financiamento pelo capital externo (GOMES, 2010). Ademais, com o fim do monopólio estatal do setor elétrico brasileiro em 1995, foi implantado um novo modelo institucional, com base na livre competição nos mercados de geração, transmissão, distribuição e comercialização de energia elétrica pelo setor privado.

Através dessas mudanças foi criada uma nova política tarifária do setor elétrico nacional contextualizado pela Agência Nacional de Energia Elétrica (ANEEL), órgão da União criado para regular e fiscalizar os novos agentes atuantes, além de realizar licitações e novas concessões e fixar os critérios de reajustes tarifários do setor elétrico. Com a nova política tarifária ocorreu mudanças na tributação, no padrão tecnológico, clima e nível de renda, nas tarifas de energia elétrica e um aumento na influência na densidade de demanda e equilíbrio econômicofinanceiro das empresas de distribuição (GOMES, 2010).

Mesmo com as mudanças nos fatores referentes à oferta de energia elétrico no Brasil, em 2001 ocorreu uma crise no setor de energia elétrica, marcante pelas condições hidrológicas extremamente desfavoráveis verificadas nas regiões Sudeste e Nordeste. O que obrigou o Governo Federal a criar a Câmara de Gestão da Crise de Energia Elétrica (GCE), com a finalidade de "propor e implementar medidas de natureza emergencial para compatibilizar a demanda e a oferta de energia elétrica, de forma a evitar interrupções intempestivas ou imprevistas do suprimento de energia elétrica" (CAMARGO, 2005). Implantando o programa de racionamento de energia elétrica, primeiramente nas regiões Sudeste, CentroOeste e Nordeste e posteriormente na Região Norte.

Nesse mesmo ano, as tarifas médias influenciadas pelo programa de racionamento reduziram o consumo de energia elétrica. O que acabaram por influenciar nas taxas de crescimento de energia elétrica. Diante desses aspectos, esse trabalho tem como objetivo estimar as elasticidades preço e renda da demanda residencial, industrial e comencial de energia elétrica nas regiões do Brasil, durante o período de 2000 a 2015, utilizando como método de estimação a ferramenta de dados em painel. Para atingir tal finalidade formalizou-se o seguinte questionamento: em que medida alterações nas tarifas de energia elétrica e na renda dos consumidores pode influenciar no consumo por energia elétrica?

Entre os trabalhos que tratam sobre o setor elétrico no âmbito nacional destacamse Andrade e Lobão (1997) que através da aplicação de um Vetor Auto Regressivo (VAR) sob a representação de um modelo de Correção de Erro (VEC) estimou a demanda residencial de energia elétrica no Brasil no período de 1963/95. Schmidt e Lima (2004) que estimaram a elasticidade renda e preço da demanda 
por energia elétrica no Brasil, nas três classes: residencial, industrial e comencial no período de 2001 a 2005, além de estimar a previsão de consumo para os próximos cinco anos posteriores ao período de estudo. Mattos (2004) que analisou a demanda por energia elétrica nos três segmentos no Estado de Minas Gerais no período de 1970 a 2002. Gomes (2010) que estimou as elasticidades preço e renda da demanda por energia elétrica residencial no Brasil no período de 1999 a 2006 por meio de painel. Modiano (1984) que estimou a elasticidade renda e preços da demanda de energia elétrica no Brasil no período de 1963 a 1981 utilizando o método dos mínimos quadrados com correção para correlação serial pelo método de Corchranne-Orcutt e Siqueira e Hollanda (2005) que calcularam a demanda setorial por energia elétrica no pós-racionamento de 2001 para o Nordeste brasileiro.

No âmbito internacional destacam-se os trabalhos de Berndt (1978) que fez uma análise sobre os estudos de Houthakker (1951). Labanderia, Labeaga e Rodriguez (2006) que estimaram a demanda por energia residencial para a Espanha. Bernstein e Griffin (2005) que estimaram as diferenças regionais na elasticidade preço da demanda por energia nos Estados Unidos. Fan e Hyndman (2010) que calcularam a elasticidade preço da demanda por eletricidade no Sul da Austrália. Reiss e White (2002) que estimularam a elasticidade preço da demanda residencial por eletricidade no Estado da Califórnia nos Estados Unidos. Casarin e Delfino (2010) que estimularam a demanda residencial por eletricidade em Buenos Aires, Filippini e Pachuari (2002) que estimularam as elasticidades por demanda por eletricidade nas residências da India. Holtedahl eJ outz (2004) que calcularam a demanda residencial por eletricidade em Taiwan e Chaudhry (2010) que calculou a elasticidade preço e renda da demanda por energia elétrica no Paquistão.

Após essa breve introdução esse trabalho está dividido em 6 seções. Na segunda seção está exposta a revisão literária, arrazoando os estudos realizados sobre as elasticidades preço e renda. Na seção 3, são apresentados o consumo de energia elétrica, no Brasil e suas regiões e nas três classes estudadas, essa seção está subdivido com a exposição histórica do PIB e PIB per capita e da tarifa de energia elétrica. Na seção posterior, está exposta a metodologia, com os dados, modelos e método de estimação das variáveis. Na seção 5 estão expostos os resultados obtidos das estimações. Na seção 6, as considerações finais e por fim as referências bibliográficas.

\section{Revisão da Literatura}

Um dos primeiros estudos realizado no Brasil sobre a elasticidade preço e renda da demanda por energia elétrica foi realizado por Modiano (1984), queatravés de dois modelos de estimação; um de ajustamento instantâneo e outro de ajustamento parcial de consumo a demanda no período de 1963 a 1981, concluiu que nas três classes analisadas: residencial, comercial e industrial, as elasticidades-preço de curto prazo foram, respectivamente - 0,118, - 0,062 e 0,451; as elasticidades-preço de longo prazo encontrado foram - 0,403, - 0,183 e - 0,222; já as elasticidades-renda de curto prazo foram 0,332, 0,362 e 0,502 e as elasticidades-renda de longo prazo foram 1,13, 1,068 e 1,360; esses resultados 
apontaram uma possível discrepância entre as tarifas relativas observadas no período e aquelas que resultariam de uma estratégia de expansão da receita setorial.

Andrade e Lobão (1997) atualizaram em parte o trabalho de Modiano (1984), utilizando um modelo econométrico para estimar a elasticidade-preço e renda da demanda residencial de energia elétrica em função também do preço dos equipamentos dos eletrodomésticos, durante o período de 1963 a 1995, as quais concluíram que as elasticidades-preço de curto e longo prazos foram respectivamente - 0,06 e - 0,051; e as elasticidades-renda de curto e longo prazos foram 0,212 e 0,213. Esses resultados demonstram o impacto que os aumentos reais nas tarifas de eletricidade teriam sobre o consumo residencial deste serviço, afetando significativamente o ritmo de crescimento da quantidade demandada do mesmo. Os autores ainda observaram outras variáveis que afetam a quantidade de consumo de energia elétrica nas residências, entre os quais, o estoque de eletrodomésticos.

Schmidt e Lima (2004) utilizando um modelo de Vetor Auto Regressivo (VAR) associado a um Modelo de Correção de Erro Vetorial (MCEV), estimaram a elasticidade preço e renda da demanda por energia elétrica no Brasil, nas três classes, durante o período de 1969 a 1999, chegando à conclusão de que, no caso residencial, o valor encontrado foi 0,539, enquanto que, nos casos comercial e industrial, os valores foram, respectivamente 0,636 e 1,916. Segundo os autores, os resultados alcançados estão de acordo com os outros resultados empíricos de quea elasticidade-renda deve ser igual ou superior à unidade e que a elasticidadepreço deve ter uma magnitude inferior a um, em módulo.

Utilizando os mesmos preceitos metodológicos para estimar a demanda por energia elétrica para o Estado de Minas Gerais nas três classes analisadas no período de 1970 a 2002. Mattos (2004) encontrou elasticidades-preço da demanda diferentes das estimadas por estudos anteriores, alcançando como resultado parâmetros estatisticamente significativos, além de seus sinais serem coerentes com a teoria econômica. Ao contrário dos estudos expostos, Gomes (2010), utilizou como parâmetro metodológico para determinar as elasticidadespreço e renda da demanda por energia elétrica residencial no Brasil no período de 1999 a 2006, um modelo de dados em painel, sugerindo um método de efeitos fixos, os valores encontrados foram - 0,111, para a elasticidade-preço e 0,091 para a elasticidade-renda, que segundo a autora os valores ficaram próximos à literatura nacional e de acordo com a teoria econômica.

Estudos internacionais sobre o tema demonstram que Holtedahl e J outz (2004) realizaram um estudo sobre a demanda residencial por energia elétrica em Taiwan de 1955 a 1996, a qual utilizando um Vetor Auto Regressivo (VAR) associado a um Modelo de Correção de Erros (MCE) atingiu que no longo prazo, a elasticidade-renda é unitária e a elasticidade-preço é inelástica e no curto prazo as elasticidades renda e preço são menores que no longo prazo. Berndt (1978) faz uma análise sobre os estudos de Houthakker (1951), a respeito das estimações da demanda total por energia elétrica em 42 províncias na Grã-Bretanha no período de 1937 a 1938.

Casarin e Delfino (2010) examinam a demanda residencial de energia elétrica na Grande Buenos Aires entre 1997 a 2006 utilizando um VAR associado a um 
VECM, a qual obteve como resultado para a elasticidade-preço no curto prazo as estimativas variam de- 0,13a- 0,63, enquanto queno longo prazo as estimativas variam de 0,16 a - 1,06, os resultados para a elasticidade-renda no curto prazo variaram entre 0,01 a 0,67 , enquanto que no longo prazo as estimativas variaram entre 0,27 a - 1,56, esses parâmetros sugerem que mudanças nas políticas de preços podem ser um instrumento eficaz para alcançar a conservação de energia elétrica em longo prazo.

Filippini e Pachauri (2002) estudaram a elasticidade preço e renda da demanda por eletricidade no setor residencial das áreas urbanas da Índia nos anos de 1993 a 1994, durante as seguintes estações: verão, inverno e monções. Utilizando um duplo modelo econométrico logarítmico linear, encontraram como resultados para a elasticidade preço e renda são inelásticas nas três temporadas estudadas.

Reiss e White (2002) estimaram a elasticidade preço e renda da demanda residencial por energia elétrica no estado americano da Califórnia de 1993 a 1997, através de um modelo GMM e OLS, resultando nos valores - 0,39 e - 0,28, para as elasticidades preço e- 0,00 e- 0,00 para as elasticidades renda. Os resultados encontrados pelos autores sugeriram uma distribuição incrivelmente distorcida da elasticidade-preço da demanda residencial.

Bernstein e Griffin (2005) analisaram a demanda por energia elétrica no uso do setor residencial de 1977 a 2004 e comercial de 1977 a 1999 e o de gás natural no setor residencial, no âmbito nacional, regional e estadual dos Estados Unidos. Utilizando um modelo de dados em painel de efeito fixo, os resultados indicam diferenças regionais e estaduais na elasticidade preço da demanda por enengia elétrica, porém tende haver alguma consistência no uso residencial de eletricidade entre os estados dentro de uma região e visíveis diferenças entre as regiões no uso de eletricidade residencial, comercial e de gás natural.

Labanderia, Labeaga e Rodriguez (2006) estimaram a demanda residencial por energia na Espanha em períodos intercalados de 1973 a 1974, de 1980 a 1981 e entre 1985 a 1995. Utilizando um modelo de extensão quadrática de Deaton e Muellbauer (1980) associado a um modelo de demanda quase ideal, proposto por Banks et al. (1997), a qual obteve como resultado que a elasticidade-preço é pequena na Espanha e a elasticidade-renda é inelástica.

Chaudhry (2010) calculou a relação de longo prazo entre a renda per capita e o crescimento previsto do consumo de eletricidade per capita e o impacto da elasticidade-preço na demanda e os insumos de energia elétrica sobre a demanda de produção de eletricidade e de produção industrial no Paquistão de 1998 a 2008, utilizando como metodologia a análise de dados em painel, a qual registrou que a elasticidade preço da demanda por eletricidade nas empresas do setor de manufatura foi de cerca de - 0,6, no do setor têxtil a elasticidade-preço foi de 0,8 e no setor de alimentos - 0,7 . O que, de acordo com o autor, significa que preços mais elevados da eletricidade irão reduzir na demanda por eletricidade no setor industrial.

\section{O Consumo de Energia Elétrica no Brasil e Regiões}


Em um período de 40 anos, o consumo total de energia elétrica no Brasil passou de 18.346 Gigawatt/ hora (GWh) na década de 1960, para 304.634 GWh em 2000, um crescimento médio anual de 7,35\%. Nas classes de estudos: residencial, comercial e industrial, essas modificações definiram diversas variações. Na década de 60, o crescimento residencial foi de 8,22\%, o comercial foi de 7,81\% e o industrial foi de $7,06 \%$. Na década seguinte, a taxa de crescimento na classe residencial foi de $10,49 \%$ ao ano, enquanto o comercial registrou um crescimento de $10,17 \%$ e o industrial foi de $13,62 \%$. Nos anos 80 , os crescimentos em comparação a década anterior, mostraram valores menores, o residencial foi de 7,62\%, o comercial registrou 5,96\% e o industrial chegou ao patamar de 5,76\% (SCHMIDT e LIMA, 2004).

Na década de 90, as taxas de crescimento anual representaram nas classes residencial, comercial e industrial, respectivamente, 5,94\%, 6,95\% e 1,94\%. No período de 2000 a 2015, o consumo final de energia elétrica no Brasil mantevese a uma taxa média de crescimento de 17,5\% ao ano (BALANÇO ENERGÉTICO NACIONAL, 2016). O consumo de energia elétrica de 1970 a 2015 no Brasil em GWh, nas três classes de consumo avaliadas: residencial, comercial e industrial ${ }^{1}$ demonstra um elevado crescimento desse recurso energético.

Figura 01 - Consumo de Energia Elétrica no Brasil (GWh)

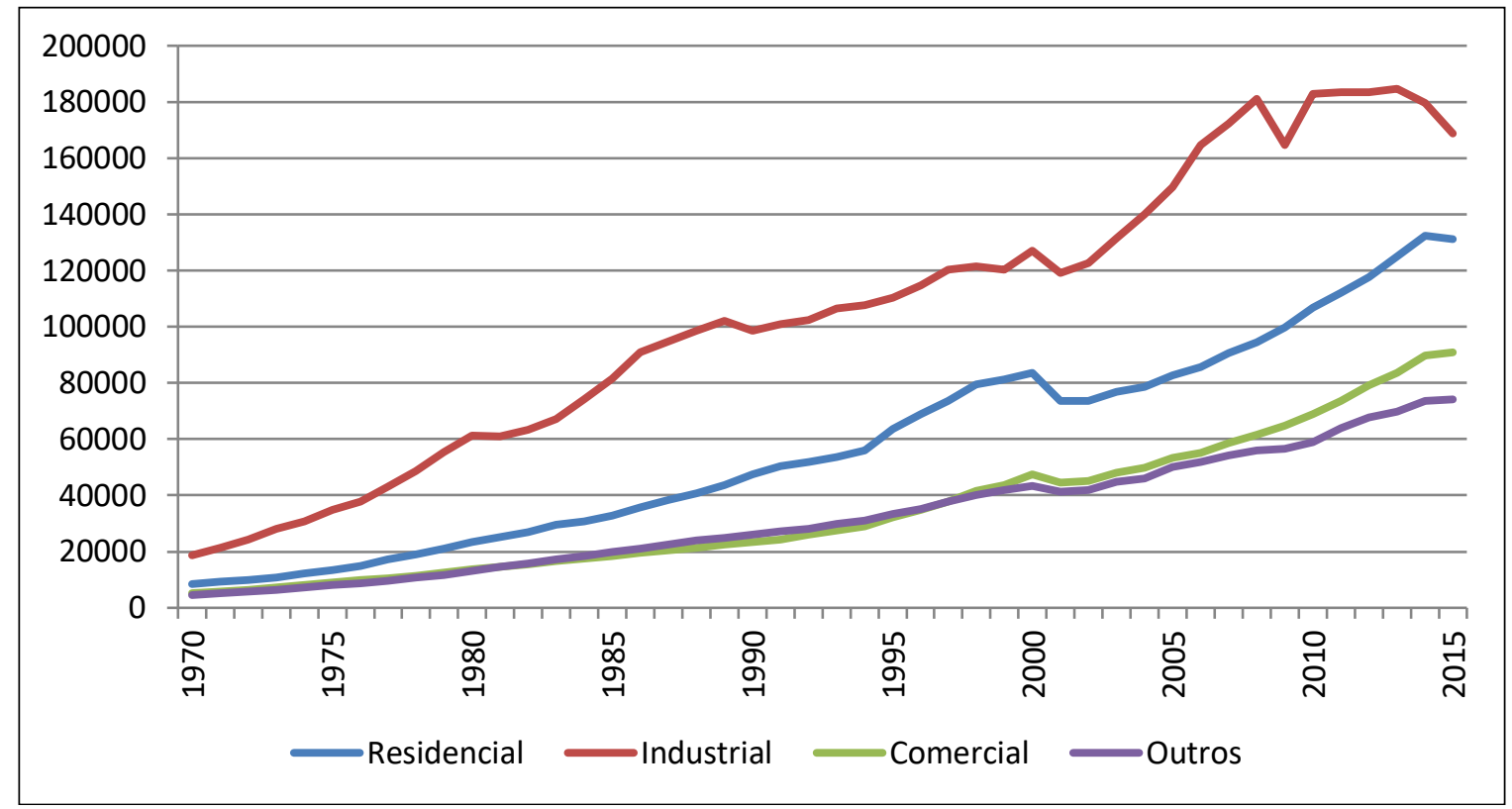

Fonte: Balanço Energético Nacional, 2016.

Elaboração: Autores.

Nas cinco regiões que compõem o Brasil, o consumo residencial de energia elétrica, mostrou índices de crescimento parecidos com o comportamento nacional, com destaque para o consumo na Região Sudeste, que apresentou crescimento bastante significativo entre 1995 e 1998, consolidando uma taxa de

\footnotetext{
${ }^{1}$ A classe de consumo denominado "outros” corresponde aos setores energéticos, público rural e transportes e não está incluída no estudo desse trabalho.
} 
crescimento anual de 7,3\%. Essa consolidação é resultado positivo da implantação do Plano Real, que permitiu um aumento do estoque eintensificação do uso de aparelhos eletroeletrônicos.

Figura 02 - Consumo Residencial de Energia Elétrica por Região no Brasil (GWh)

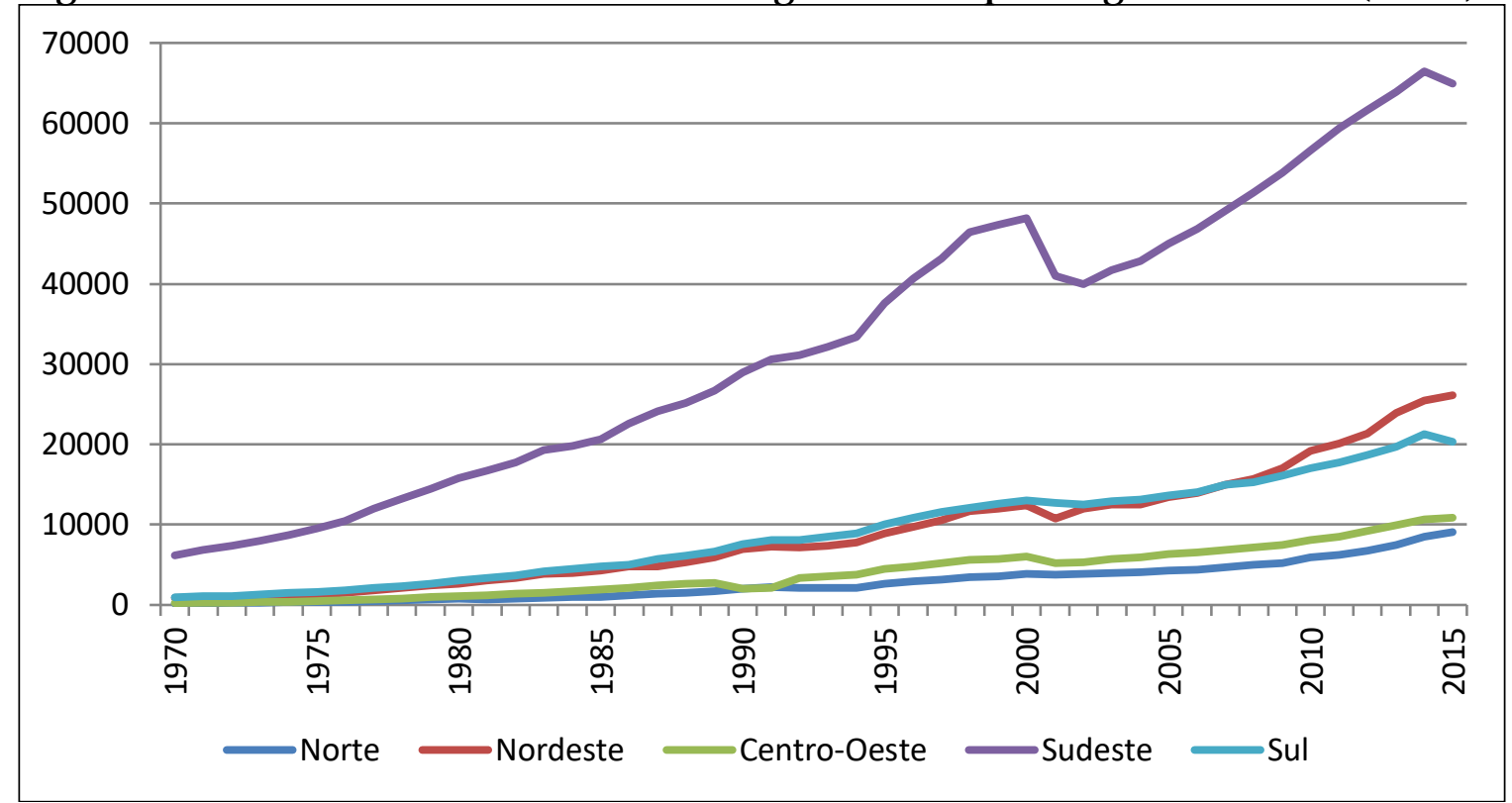

Fonte: Empresa de Pesquisa Energética, 2016.

Elaboração: Autores.

A classe de consumo comercial demonstrou entre 1970 a 2015 um aumento gradativo no mercado ao final da década de 80 , sendo a Região Sudeste com maior representatividade, com crescimento entre 1980 a 1990 de $11 \%$ ao ano; em 1995 esse crescimento passou para 13\%; em 2000 esse valor aumentou para 16\%; enquanto que em 2004 atingiu os 17\% ao ano. A Região Nordeste foi o que mais cresceu, após a Região Sudeste no consumo comercial de energia elétrica, devido aos incentivos estaduais na construção de parques aquáticos, complexos hoteleiros e polos de ecoturismo, favorecendo a demanda por essa fonte de 
energia. Esse crescimento foi interrompido em 2001, durante o racionamento de energia.

Figura 03 - Consumo Comercial de Energia Elétrica por Região no Brasil (GWh)

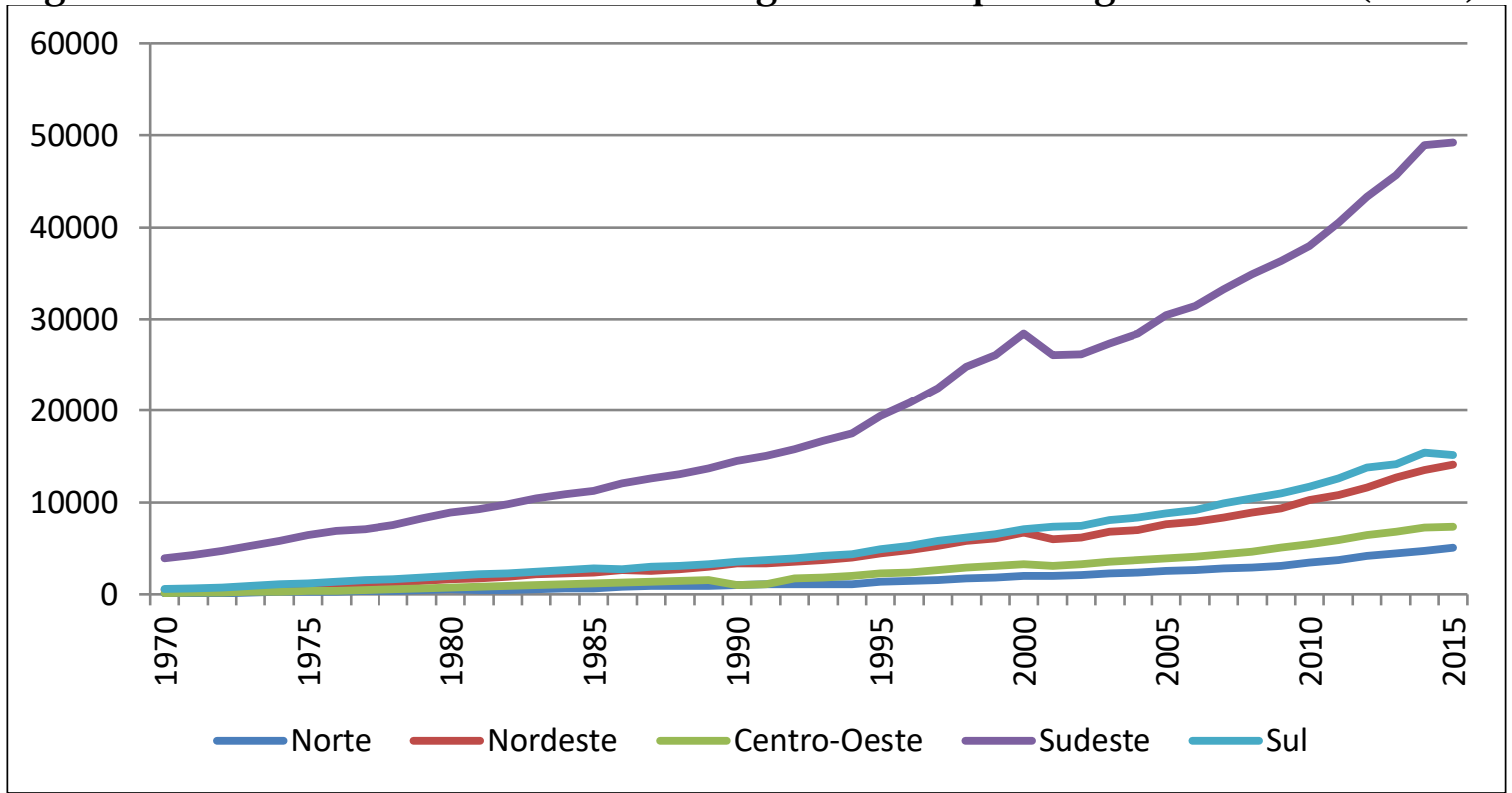

Fonte: Empresa de Pesquisa Energética, 2016.

Elaboração: Autores.

O consumo por energia elétrica no setor industrial nas regiões brasileiras demonstra um comportamento bastante singular, a concentração da demanda em uma única região - Sudeste, o que contrapõem o ocorrido com a Região Norte que só apresentou forte expansão entre 1980 e 1990 com a implantação da Zona Franca de Manaus e da Usina de Tucuruí, que associado posteriormente a indústria de mineração, alavancou o consumo industrial por energia elétrica nessa região. Na Região Nordeste, o impulso se deu com a criação do complexo petroquímico de Camaçari na Bahia, no final da década de 70, que associado ao complexo de alumínio no Maranhão e das lavouras de fruticultura irrigada e outros ramos da indústria têxtil, de bebidas e alimentar, alavancou o consumo nessa região.

A Região Sudeste concentra o maior número de indústrias no Brasil, como consequência apresenta o maior consumo por energia elétrica nesse setor da economia; a região da Grande São Paulo abrange o maior parque industrial da América Latina, o Estado de Minas Gerais é o segundo maior centro industrial do país e o Estado do Rio de J aneiro possui na expansão da indústria petrolífera seu grande desempenho na economia dessa região. Entre as regiões, o Centro-Oeste é o que possui menor participação no consumo industrial de energia elétrica, já que sua economia está voltada principalmente ao setor agropecuário e de turismo ambiental. Assim como a Região Sudeste, a Região Sul foram as que mais sentiram os efeitos ocorridos na economia durante o período exposto, com quedas no consumo, devido aos racionamentos instituídos no início da década de 80 e em 2001 e a crise internacional de 2008/2009.

Figura 04 - Consumo Industrial de Energia Elétrica por Região no Brasil (GWh) 


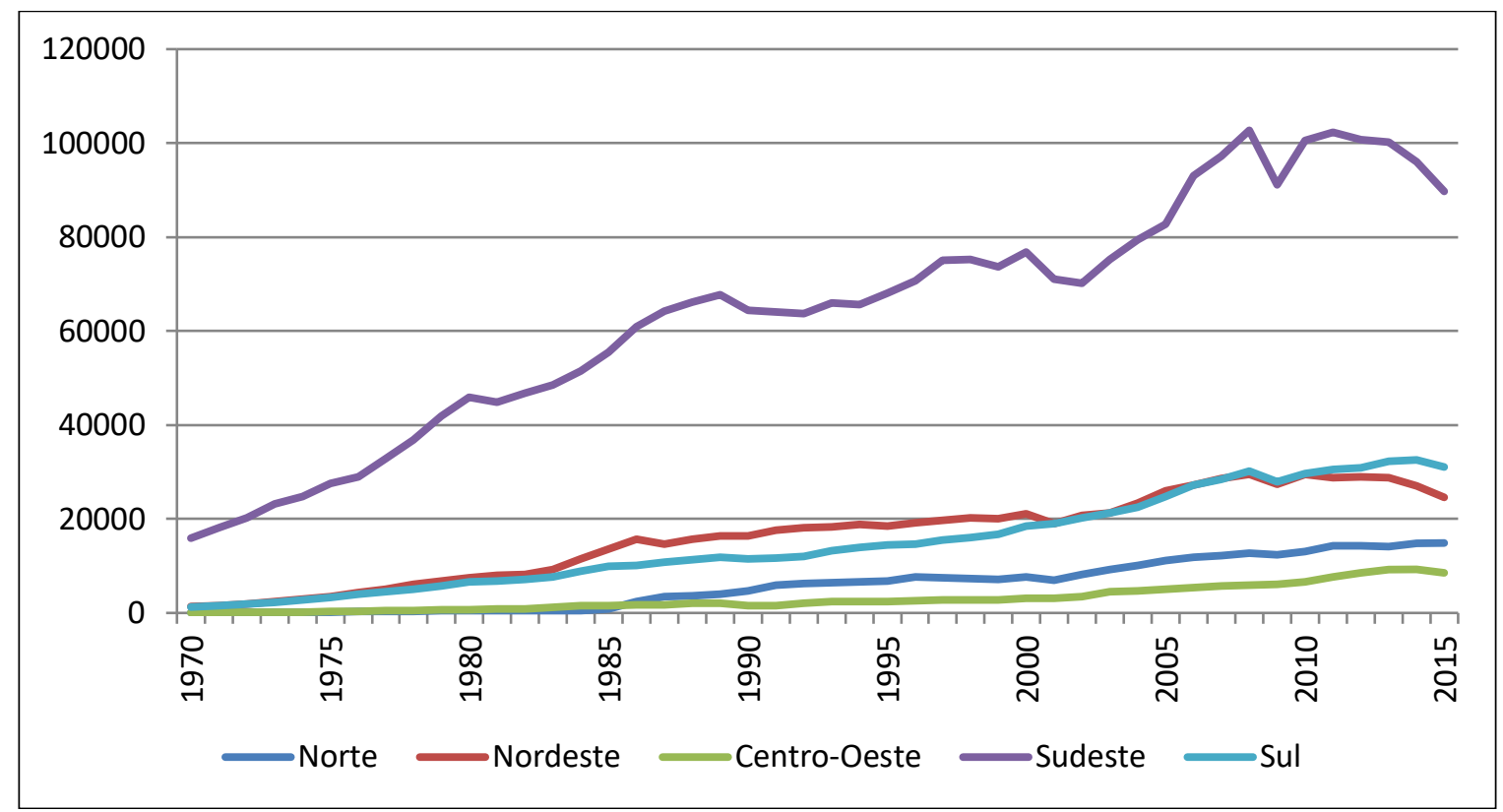

Fonte: Empresa de Pesquisa Energética, 2016.

Elaboração: Autores.

3.1Tarifas de Energia Elétrica

Na década de 70, as tarifas de energia elétrica no Brasil passaram por variações positivas ocorridas pelo aumento real nas taxas no início da década, porém essa situação não ocorreu nas décadas posteriores. Novos aumentos reais só ocorreram novamente a partir da década de 90. Entre as classes de consumo: residencial, industrial e comercial, os aumentos registrados na década de 70, merecem destaque os ocorridos em 1972 e 1975. As reduções ocorridas durante a década de 80, mais precisamente entre os anos de 1980 e 1989, nas tarifas residencial, comercial e industrial tiveram quedas de $42,46 \%$, 20,68\% e 13,47\%, respectivamente (SCHMIDT e LIMA, 2004).

Segundo Schmidt e Lima (2004), as tarifas residencial, comercial e industrial tiveram os mais elevados níveis de preços nos anos de 1972, 1972 e 1965, respectivamente, com valores de 338,73; 332,21 e 128,50, e os anos que registraram os menos valores nas tarifas das classes residencial, comercial e industrial foram os de 1986, 1985 e 1985, com níveis de preços de 78,92; 119,53 e 64,55 , respectivamente. Os valores percentuais registrados, nesses anos de elevados níveis de preços e de baixos valores de cada classe estudada foram de $76,70 \%,-64,02 \%$ e - 49,77\%, respectivamente.

Entre 1990 a 2000, ocorreram leves crescimentos nas tarifas das classes estudadas, contudo entre 2001 e 2007, as tarifas de energia elétrica no Brasil cresceram em média de R\$ 122,88 para R\$ 260,48 por Megawatt-hora (MW/h), recuando nos anos posteriores. Na classe residencial entre 2001 a 2010, os valores das tarifas registraram $\mathrm{R} \$ 179,78$ e $\mathrm{R} \$ 300,07 \mathrm{MWh}$, respectivamente. No que condiz da classe comercial o resultado no mesmo período foi de $\mathrm{R} \$ 156,17$ e R \$287,05 por Megawatt-hora. Nesse mesmo período, os valores das tarifas médias anuais da classe industrial contabilizaram R\$ 81,18 e R\$ 236,46 MWh. 
As tarifas que apresentaram as maiores variações foram às relativas às classes residencial e comercial. Comparando os níveis de preços entre os anos de 1970 e 1979, a tarifa residencial decaiu em 20,48 \%, a tarifa comercial 21,13 \% e a industrial 17,27\%. Na década de 1980, entre os anos de 1980 e 1989, as tarifas residencial, comercial e industrial tiveram uma queda de 42,46 \%, 20,68 \% e $13,47 \%$, concomitantemente, sugerindo a persistência da queda real das tarifas no setor energético. No período compreendido de 1990 e 2000, ocorreu um aumento real nas tarifas da classe de consumo residencial de 14,69 \%, enquanto que nas classes de consumo comercial foi de - 22,78 \% e a na classe industrial foi de - 21, 61\% (SCHMIDT e LIMA, 2004).

Em 2001, as tarifas médias, influenciadas pelo programa de redução do consumo que havia sido criado como solução de curto prazo para a crise energética, tiveram um aumento significativo em termos reais, visando recuperar as receitas das empresas do setor energético (MATTOS, 2004).

\section{Metodologia}

Os dados utilizados na realização da estimação da demanda por energia elétrica são anuais e com periodicidade de 2000 a 2015. A fonte dos dados foi retirada de diversas referências, entre as quais a Empresa de Pesquisa Energética (EPE), a Eletrobrás, o Instituto Brasileiro de Geografia e Estatística (IBGE) e a Fundação Getúlio Vargas (FGV). A identificação das fontes dos dados das variáveis deste trabalho está exposta na Tabela 01, a seguir.

Tabela 01 - Identificação das Fontes dos Dados das Variáveis

\begin{tabular}{c|c|c|c}
\hline $\begin{array}{c}\text { Variáveis } \\
\text { (Séries } \\
\text { anuais de } \\
\text { 2000 a 20 15) }\end{array}$ & Proxy Utilizada & Fontes & Unidade \\
\hline \multicolumn{4}{c}{ Classe Residencial } \\
\hline $\mathrm{Y}$ & Consumo Residencial Total & $\mathrm{EPE}$ & $\mathrm{GWh}$ \\
\hline $\mathrm{T}$ & $\begin{array}{c}\text { Tarifa Média Residencial em } \\
\text { Valores de Mercado }\end{array}$ & ANEEL & $\mathrm{R} \$ / \mathrm{MWh}$ \\
\hline $\mathrm{P}$ & $\begin{array}{c}\text { IPA-DI - Bens de Consumo } \\
\text { Duráveis }\end{array}$ & $\begin{array}{c}\text { Banco de Dados } \\
\text { IBRE/FGV/ RJ }\end{array}$ & İndice \\
\hline $\mathrm{R}$ & $\begin{array}{c}\text { PIB per capita a preços de } \\
\text { mercado }\end{array}$ & IBGE & $\mathrm{R} \$$ \\
\hline $\mathrm{Y}$ & Classe Comercial \\
\hline $\mathrm{T}$ & Tarifa Média Comercial em \\
Valores de Mercado & ANEEL & $\mathrm{R} \$ / \mathrm{MWh}$ \\
\hline $\mathrm{P}$ & IPA-OG - Material Elétrico & $\begin{array}{c}\text { Banco de Dados } \\
\text { IBRE } / \mathrm{FGV} / \mathrm{RJ}\end{array}$ & İndice \\
\hline $\mathrm{R}$ & PIB: Serviços & IBGE & $\mathrm{R} \$$ \\
\hline
\end{tabular}

\section{Classe Industrial}




\begin{tabular}{|c|c|c|c|}
\hline $\mathrm{Y}$ & Consumo Industrial Total & EPE & GWh \\
\hline $\mathrm{T}$ & $\begin{array}{c}\text { Tarifa Média Industrial em } \\
\text { Valores de Mercado }\end{array}$ & ANEEL & $\mathrm{R} \$ / \mathrm{MWh}$ \\
\hline $\mathrm{P}$ & $\begin{array}{l}\text { IPA-OG - Máquinas e } \\
\text { Equipamentos Industriais }\end{array}$ & $\begin{array}{l}\text { Banco de Dados } \\
\text { IBRE/FGV/RJ }\end{array}$ & Índice \\
\hline $\mathrm{R}$ & PIB Industrial & IBGE & $\mathrm{R} \$$ \\
\hline TB & $\begin{array}{c}\text { IPA-OG - Combustíveis e } \\
\text { Lubrificantes }\end{array}$ & $\begin{array}{l}\text { Banco de Dados } \\
\text { IBRE/ FGV/ RJ }\end{array}$ & Índice \\
\hline
\end{tabular}

Fonte: Elaborado pelos autores.

As estatísticas descritivas das variáveis aplicadas a esse trabalho estão distribuídas entre as variáveis: Consumo Total $\left(Y_{i t}\right)$, Tarifa Média $\left(T_{i t}\right)$, Índice de Preço $\left(P_{i t}\right)$ e Renda/PIB $\left(R_{i t}\right)$ para todas as classes de consumo, adicionando a classe de consumo industrial a variável Índice de Preço do Bem Substituto $\left(T B_{i t}\right)$

Tabela 02 - Estatísticas Descritivas das Variáveis Aplicadas

\begin{tabular}{c|c|c|c|c|c}
\hline \multicolumn{5}{c}{ Consumo Residencial } & \multicolumn{2}{c}{ Amplitude } \\
\hline $\begin{array}{c}\text { Variávei } \\
\text { S }\end{array}$ & Média & $\begin{array}{c}\text { Desvio } \\
\text { Padrão }\end{array}$ & Mediana & Mínimo & Máximo \\
\hline$Y_{i t}$ & $19.552,02$ & $17.532,22$ & $13.001,5$ & 3.740 & 66.458 \\
\hline$T_{i t}$ & 274,95 & 66,69 & 287,23 & 131,10 & 454,01 \\
\hline$P_{i t}$ & 410,76 & 102,47 & 407,32 & 243,91 & 555,09 \\
\hline$R_{i t}$ & $15.961,41$ & $9.459,50$ & $13.998,47$ & $3.075,45$ & $39.045,21$ \\
\hline
\end{tabular}

Consumo Comercial

\begin{tabular}{|c|c|c|c|c|c|}
\hline$Y_{i t}$ & $12.684,53$ & $12.387,42$ & $7.519,00$ & 1.974 & 49.223 \\
\hline$T_{i t}$ & 265,86 & 66,17 & 279,64 & 111,70 & 437,93 \\
\hline P. & 209,08 & 35,36 & 231,87 & 131,55 & 238,51 \\
\hline$R_{i t}$ & $\begin{array}{c}34.983 .13 \\
1\end{array}$ & $\begin{array}{c}38.722 .74 \\
8\end{array}$ & 20.738 .653 & $\begin{array}{c}29.419 .50 \\
0\end{array}$ & 175.093 .302 \\
\hline
\end{tabular}

\section{Consumo Industrial}

\begin{tabular}{c|c|c|c|c|c}
\hline$Y_{i t}$ & $31.902,63$ & $30.355,76$ & $21.889,50$ & 3.054 & 102.717 \\
\hline$T_{i t}$ & 196,50 & 72,01 & 215,45 & 40,90 & 397,41 \\
\hline$T B_{i t}$ & 89,17 & 37,88 & 73,66 & 48,43 & 150,54 \\
\hline$P_{i t}$ & 87,40 & 30,05 & 81,31 & 47,49 & 141,69 \\
\hline$R_{i t}$ & 13.631 .353 & $\begin{array}{c}15.238 .93 \\
8\end{array}$ & $\begin{array}{c}68.032 .88 \\
0\end{array}$ & 12.233 .307 & $\begin{array}{c}509.029 .40 \\
4\end{array}$ \\
\hline
\end{tabular}

Fonte: Elaborado pelos autores.

Nos diversos estudos sobre a elasticidade preço e renda da demanda de energia elétrica existente, os cálculos utilizados partem de um modelo padrão, as quais 
utilizam como variáveis nas equações, alguns fatores determinantes para cada classe de consumo. Na demanda na classe residencial pode ser proposta como o resultado de um problema de maximização da utilidade, sujeita a uma restrição orçamentária. Nas classes comerciais e industriais pode ser descrito como um problema de minimização de custos, sujeito a certo nível de produção (SCHMIDT e LIMA, 2004).

Nesse caso utilizam-se como variáveis para determinar a função demanda por energia elétrica para qualquer classe de consumo: o consumo por energia elétrica no tempo $\left(Y_{t}\right)$, a tarifa de energia elétrica no tempo $\left(T_{t}\right)$, a tarifa do bem substituto a energia elétrica no tempo $\left(T B_{t}\right)$, a renda da classe em questão no tempo $\left(R_{t}\right)$ e o preço dos aparelhos ou máquinas elétricas que constitui cada classe de consumo no tempo $\left(P_{t}\right)$.

Algebricamente a equação de demanda por energia elétrica é descrita de forma linear da seguinte maneira:

$$
Y_{t}=\beta_{0} Y_{t-1}+\beta_{1} T_{t}+\beta_{2} T B_{t}+\beta_{3} R_{t}+\beta_{4} P_{t}+\alpha Z_{i}+\varepsilon_{t}
$$

Contudo, para se encontrar a elasticidade das variáveis em questão faz-se necessário pôr a equação acima na forma logarítmica:

$$
\ln Y_{t}=\beta_{0} \ln Y_{t-1}+\beta_{1} \ln T_{t}+\beta_{2} \ln T B_{t}+\beta_{3} \ln R_{t}+\beta_{4} \ln P_{t}+\alpha Z_{i}+\varepsilon_{t}
$$

Onde: $\beta_{1}, \beta_{2}, \beta_{3}$ e $\beta_{4}$ são as elasticidades das variáveis da equação, $Z_{i}$ representam as dummies para cada região e $\varepsilon_{t}$ é o erro estacionário.

Para estimar o modelo as elasticidades - preço e renda da demanda por energia elétrica nas regiões do Brasil será utilizado o modelo de regressão conhecido como dados em painel.

Os dados em painel são modelos estabelecidos para estimar parâmetros através de um conjunto de amostras longitudinais com dimensão de corte transversal e temporal. A utilização dessa ferramenta tem como vantagem o fornecimento de uma maior quantidade de informações, diversidade de comportamentos individuais, maior variabilidade dos dados, menor colinearidade entre as variáveis, maior número de graus de liberdade e maior eficiência na estimação. Dessa forma, permite identificar, controlar e medir efeitos individuais que não serão pura e simplesmente detectáveis em estudos exclusivamente seccionais ou temporais, bem como estabelecer e testar modelos comportamentais complexos, especificamente recorrendo a modelos com defasagens distribuídas com poucas restrições (MARQUES, 2000).

A estrutura básica de dados em painel é um modelo de regressão da forma:

$$
Y_{i t}=X_{i t} \beta+Z_{i} \alpha+\varepsilon_{i t}
$$

Em que $X_{i t}$ possui $\mathrm{K}$ regressores, incluindo um termo constante; $Z_{i} \alpha$ é a heterogeneidade ou o efeito individual, onde $Z_{\mathrm{i}}$ contém um termo constante e um 
conjunto de individuo ou grupo de variáveis específicas, que podem ser observadas ou não, tais como raça, sexo, localização, características especificas de família, habilidades ou preferências e assim por diante, os quais são levados para ser constantes ao longo do tempo t.

O modelo dinâmico com dados em painel utiliza-se do valor defasado da variável resposta $Y_{i t-1}$ para determinar as variáveis regressoras, através da seguinte equação:

$$
\begin{gathered}
Y_{i t}=\delta Y_{i t-1}+X_{i t} \beta+Z_{i} \alpha+\varepsilon_{i t} \\
\varepsilon_{i t}=\theta_{i}+\eta_{i t}
\end{gathered}
$$

Em que $i=1, \ldots, N$ e $t=2, \ldots, T$, onde $\theta_{i}+\eta_{i t}$ é o usual componente do erro da decomposição do termo do erro, $\mathrm{N}$ é extenso, $\mathrm{T}$ é fixo e $|\delta|<1$. Esse modelo específico é suficiente para cobrir a maior parte dos casos padrão encontrado nas aplicações linear de dados em painel dinâmico. Permitindo que a inclusão de $X_{i t-1}$ providencie um modelo dinâmico de dados em painel auto-regressivo.

$$
Y_{i t}=\delta Y_{i t-1}+X_{i t} \beta_{1}+X_{i t-1} \beta_{2}+Z_{i} \alpha+\theta_{i}+\eta_{i t}
$$

Que tem um correspondente fator restrito comum $\left(\beta_{2}=-\delta \beta_{1}\right)$, o que resulta em:

$$
y_{i t}=+X_{i t} \beta_{1}+\varpi_{i}+\vartheta_{i t}
$$

Com $\vartheta_{i t}=\delta \vartheta_{i t-1}+\eta_{i t}$ e $\theta_{i}=(1-\delta) \varpi_{i}$. Um dos problemas com a estimação de modelos dinâmicos com dados em painel comum aos diversos estudos realizados é a endogeneidade, ou seja, a correlação existente entre um dos regressores $Y_{i t-1}$ e o termo do erro $\varepsilon_{i t}$ através de $\theta_{i}$. Essa situação torna os estimadores de Mínimos Quadrados Ordinários (MQO) enviesados e não consistentes, mesmo que $\eta_{i t}$ não demonstre autocorrelação, podendo o enviesamento assintótico ser significativo (MARQUES, 2000). Para corrigir o problema de perda de consistência dos estimadores convencionais dos modelos dinâmicos com dados em painel, utilizase o Método dos Momentos Generalizados (GMM), em uma versão conhecida como Difference-GMM. Que possui como objetivo o de encontrar um estimador consistente com um mínimo de restrições sobre os momentos. Logo, a equação de estimação do modelo utilizado passa a ser a seguinte:

$$
\begin{gathered}
\Delta \ln Y_{i t}=\beta_{0} \Delta \ln Y_{i t-1}+\beta_{1} \Delta \ln T_{i t}+\beta_{2} \Delta \ln T B_{i t}+\beta_{3} \Delta \ln R_{i t}+\beta_{4} \Delta \ln P_{i t}+\alpha \Delta Z_{i} \\
+\Delta \varepsilon_{i t}
\end{gathered}
$$

Mesmo após essa transformação, o problema da endogeneidade ainda persiste, já que $\Delta \varepsilon_{i t}$ são correlacionados com $\Delta \ln Y_{i t-1}$. Para resolver definitivamente esse problema, Arellano e Bond (1991) recomendam utilizar a first-differenced (primeira diferença), que consiste em retirar as primeiras diferenças de todas as variáveis do modelo, para eliminar o problema da endogeneidade. 
Contudo, se o painel possui uma dimensão temporal pequena, o uso da firstdifferenced não resolverá o problema da endogeneidade. Sendo assim, a utilização do modelo System-GMM, a qual emprega duas técnicas de estimação, com base nas defasagens, formando duas equações, a original ou equação de nível, que utiliza as defasagens em diferença como instrumentos; e a equação transformada ou em diferença, que utiliza as variáveis defasadas em nível como instrumentos (Arellano e Bover, 1995; Blundell e Bond, 1998).

Os estimadores Arellano-Bond (1991) e Blundell-Bond (1998) possuem dois variantes o one-step e o two-step. Sendo a segunda variante (two-step) assintoticamente mais eficiente, porém, os erros-padrão reproduzidos nessa ocasião possuem uma tendência de serem severamente viesados para baixo. Sendo assim, utiliza-se uma correção de amostras finitas, derivadas por Windmeijer (2005), para a matriz de covariância da variante two-step, o que transforma as estimações robustas da variante two-step mais eficientes do que as estimações robustas da variante one-step.

Para testar a validade das condições de momento inerentes ao GMM, adotam-se as estatísticas de teste de Sargan (1958) e Hansen (1982), que possui como hipótese nula a validade dos instrumentos, ou seja, não correlacionados com o termo erro e que os instrumentos excluídos (aqueles utilizados para instrumentar as variáveis endógenas) foram corretamente excluídos da equação estimada. A estatística Sargan é um caso especial da J de Hansen pela suspeita de homoscedasticidade. A estatística de Hansen é utilizada pela suspeita de erros heteroscedásticos.

\section{Resultados}

As estimações das elasticidades preço e renda demanda foram realizadas pelo modelo de dados em painel, utilizando a metodologia System-GMM, que foi empregada nessa avaliação, devido sua eficácia na eliminação do viés que ocorre no painel dinâmico. Os resultados são apresentados na tabela 3 abaixo, e foram divididos em consumo residencial, consumo comercial, e consumo industrial.

Tabela 03 - Resultado dos Modelos de Regressões para Determinar as Elasticidades-Preço e Renda

\begin{tabular}{c|c|c|c|c|c}
\hline & \multicolumn{5}{|c}{ Classe de Consumo Residencial } \\
\hline $\begin{array}{c}\text { Parâmetro de } \\
\text { Elasticidades }\end{array}$ & Norte & Nordeste & $\begin{array}{c}\text { Centro- } \\
\text { Oeste }\end{array}$ & Sudeste & Sul \\
\hline & $-0,2751$ & $-0,1527$ & $-0,1671$ & $-0,2360$ & $-0,1965$ \\
$\begin{array}{c}\text { Tarifa Residencial } \\
\left(\mathrm{T}_{\mathrm{t}}\right)\end{array}$ & $(0,0563)^{*}$ & $(0,0350)$ & $(0,0357)$ & $(0,0125)$ & $(0,0345)$ \\
\hline $\begin{array}{c}\text { Preço de } \\
\begin{array}{c}\text { Eletrodomésticos } \\
\left(\mathrm{P}_{\mathrm{t}}\right)\end{array}\end{array}$ & $-0,2351$ & $-0,2151$ & $-0,2755$ & $-0,6431$ & $-0,3510$ \\
\hline
\end{tabular}




\begin{tabular}{|c|c|c|c|c|c|}
\hline $\begin{array}{c}\text { Renda Familiar } \\
\left(\mathrm{R}_{\mathrm{t}}\right)\end{array}$ & $\begin{array}{c}0,2145 \\
(0,0241)\end{array}$ & $\begin{array}{c}0,1930 \\
(0,0391)\end{array}$ & $\begin{array}{c}0,3681 \\
(0,0492)\end{array}$ & $\begin{array}{c}0,1954 \\
(0,0322)\end{array}$ & $\begin{array}{c}0,0432 \\
(0,0266)\end{array}$ \\
\hline & \multicolumn{5}{|c|}{ Classe de Consumo Comercial } \\
\hline $\begin{array}{l}\text { Parâmetro de } \\
\text { Elasticidades }\end{array}$ & Norte & Nordeste & $\begin{array}{c}\text { Centro- } \\
\text { Oeste }\end{array}$ & Sudeste & Sul \\
\hline $\begin{array}{l}\text { Tarifa Comercial } \\
\qquad\left(\mathrm{T}_{\mathrm{t}}\right)\end{array}$ & $\begin{array}{l}-0,2854 \\
(0,0218)\end{array}$ & $\begin{array}{l}-0,5321 \\
(0,0123)\end{array}$ & $\begin{array}{l}-0,2911 \\
(0,0331)\end{array}$ & $\begin{array}{l}-0,1967 \\
(0,0291)\end{array}$ & $\begin{array}{l}-0,2991 \\
(0,0218)\end{array}$ \\
\hline $\begin{array}{l}\text { Preço de Material } \\
\text { Elétrico }\left(\mathrm{P}_{\mathrm{t}}\right)\end{array}$ & $\begin{array}{l}-0,0521 \\
(0,0655)\end{array}$ & $\begin{array}{l}-1,0981 \\
(0,0283)\end{array}$ & $\begin{array}{l}-0,3305 \\
(0,0802)\end{array}$ & $\begin{array}{l}-0,0145 \\
(0,0871)\end{array}$ & $\begin{array}{l}-0,2235 \\
(0,0452)\end{array}$ \\
\hline \multirow{2}{*}{$\begin{array}{c}\text { PIB Comercial } \\
\left(\mathrm{R}_{\mathrm{t}}\right)\end{array}$} & $\begin{array}{c}0,1959 \\
(0,0194)\end{array}$ & $\begin{array}{c}0,1794 \\
(0,0258)\end{array}$ & $\begin{array}{c}0,2922 \\
(0,0150)\end{array}$ & $\begin{array}{c}0,0369 \\
(0,0246)\end{array}$ & $\begin{array}{c}0,0722 \\
(0,0213)\end{array}$ \\
\hline & \multicolumn{5}{|c|}{ Classe de Consumo Industrial } \\
\hline $\begin{array}{l}\text { Parâmetro de } \\
\text { Elasticidades }\end{array}$ & Norte & Nordeste & $\begin{array}{c}\text { Centro- } \\
\text { Oeste }\end{array}$ & Sudeste & Sul \\
\hline $\begin{array}{c}\text { Tarifa Industrial } \\
\left(\mathrm{T}_{\mathrm{t}}\right)\end{array}$ & $\begin{array}{l}-0,1532 \\
(0,0599)\end{array}$ & $\begin{array}{l}-0,0912 \\
(0,0430)\end{array}$ & $\begin{array}{l}-0,0736 \\
(0,0125)\end{array}$ & $\begin{array}{l}-0,2145 \\
(0,0451)\end{array}$ & $\begin{array}{l}-0,0652 \\
(0,0493)\end{array}$ \\
\hline $\begin{array}{c}\text { Preço de } \\
\text { Máquinas e } \\
\text { Equipamentos } \\
\text { Industriais }\left(\mathrm{P}_{\mathrm{t}}\right)\end{array}$ & $\begin{array}{c}-0,5903 \\
(0,0421)\end{array}$ & $\begin{array}{l}-1,3557 \\
(0,4219)\end{array}$ & $\begin{array}{l}-1,2791 \\
(0,0844)\end{array}$ & $\begin{array}{l}-0,3901 \\
(0,0199)\end{array}$ & $\begin{array}{c}-0,5902 \\
(0,1132)\end{array}$ \\
\hline $\begin{array}{c}\text { Preço do Bem } \\
\text { Substituto a } \\
\text { Energia Elétrica } \\
\left(\mathrm{TB}_{\mathrm{t}}\right)\end{array}$ & $\begin{array}{c}0,1892 \\
(0,0289)\end{array}$ & $\begin{array}{c}0,5338 \\
(0,1870)\end{array}$ & $\begin{array}{c}0,8447 \\
(0,0193)\end{array}$ & $\begin{array}{c}0,0521 \\
(0,0392)\end{array}$ & $\begin{array}{c}0,1823 \\
(0,0432)\end{array}$ \\
\hline $\begin{array}{c}\text { PIB Industrial } \\
\left(\mathrm{R}_{\mathrm{t}}\right)\end{array}$ & $\begin{array}{c}0,6819 \\
(0,0392)\end{array}$ & $\begin{array}{c}0,6721 \\
(0,2812)\end{array}$ & $\begin{array}{c}0,2381 \\
(0,0234)\end{array}$ & $\begin{array}{c}0,1341 \\
(0,0285)\end{array}$ & $\begin{array}{c}0,1429 \\
(0,0326)\end{array}$ \\
\hline \multicolumn{3}{|c|}{$\begin{array}{l}\text { H0: Ausência de Autocorrelação dos resíduos } \\
\text { de primeira ordem }\end{array}$} & \multicolumn{2}{|l|}{ p-valor } & 0,00 \\
\hline \multicolumn{3}{|c|}{$\begin{array}{l}\text { H0: Ausência de Autocorrelação dos resíduos } \\
\text { de segunda ordem }\end{array}$} & \multicolumn{2}{|l|}{ p-valor } & 0,16 \\
\hline \multicolumn{3}{|c|}{ Teste de Sargan } & \multicolumn{2}{|l|}{ Prob > Chi2 } & 0,1281 \\
\hline \multicolumn{3}{|c|}{ Teste de Hansen } & \multicolumn{2}{|l|}{ Prob > Chi2 } & 0,3821 \\
\hline \multicolumn{3}{|c|}{ Teste Difference-Hansen } & \multicolumn{2}{|l|}{ Prob > Chi2 } & 0,1204 \\
\hline
\end{tabular}


Fonte: Elaborado pelos autores.

*Os valores entre parênteses representam os erros padrões das amostras; Estimativas significantes em nível de $5 \%$.

Consumo Residencial

A Tabela 03 acima mostra que as elasticidades-preço apresentaram sinais negativos, enquanto que a elasticidade-renda apresentou sinal positivo, indicando que quanto maior o preço da tarifa e dos eletrodomésticos menos a demanda por energia elétrica, e na outra mão, quanto maior a renda maior a demanda por energia elétrica. Ademais, a Região Norte apresentou a maior elasticidade-preço da demanda da tarifa residencial (que foi de - 0,2751). Por outro lado, a Região Nordeste foi a que apresentou a menor elasticidade-preço da demanda da tarifa residencial (que foi de - 0,1527), além disso, a Região Sul apresentou menor elasticidade-renda da demanda (que foi de 0,0432), enquanto que a Região Centro-Oeste apresentou a maior elasticidade-renda da demanda (que foi de 0,3681). Já a Região Sudeste obteve a maior elasticidade-preço da demanda de eletrodomésticos (que foi de - 0,6431), por outro lado, obteve a segunda maior elasticidade preço da demanda da tarifa residencial (que foi de $0,2360)$.

\section{Consumo Comercial}

O resultado da Tabela 03 mostra que as elasticidades-preço apresentaram sinais negativos, sugerindo que quanto maior o preço da tarifa e de materiais elétrico menor a demanda por energia elétrica, enquanto que as elasticidades-renda apresentaram sinal positivo, indicando que quanto maior a renda, maior é a demanda por energia elétrica. Ademais, a Região Nordeste apresentou as maiores elasticidades-preço da demanda da tarifa comercial (que foi de - 0,5321) e da demanda de material elétrico (que foi de - 1,0981), enquanto que a Região Centro-Oeste exibiu a maior elasticidade-renda da demanda (que foi de 0,2922). Por outro lado, a Região Sudeste apresentou as menores elasticidade-preço da demanda da tarifa comercial (que foi de - 0,1967), elasticidade-preço da demanda de material elétrico (que foi de - 0,0145), e elasticidade-renda da demanda (que foi de 0,0369), além disso, a Região Sul apresentou a segunda maior elasticidade-preço da demanda da tarifa comercial (que foi de - 0,2991) e a segunda menor elasticidade-renda da demanda (que foi de 0,0722).

\section{Consumo Industrial}

A Tabela 03 acima mostra que as elasticidades-preço da tarifa e de máquinas e equipamentos industriais exibiram sinais negativos, sugerindo que quanto maior o preço desses parâmetros menor a demanda por energia elétrica, contraposto a isso, as elasticidade-preço do bem substituto a energia elétrica e a elasticidaderenda apresentaram sinais positivos, sugerindo que quanto maior o preço e a renda, maior a demanda por energia elétrica. Além do mais, a Região Nordeste apresentou a maior elasticidade-preço da demanda de máquinas e equipamentos industriais (que foi de - 1,3557) e a segunda maior da elasticidade-renda da demanda (que foi de 0,6721), enquanto que a Região Sudeste apresentou a maior 
elasticidade-preço da demanda da tarifa industrial (que foi de - 0,2145) e a Região Norte apresentou a maior elasticidade-renda da demanda (que foi de $0,6819)$. Por outro lado, a Região Sudeste apresentou as menores elasticidadespreço da demanda de máquinas e equipamentos industriais (que foi de - 0,3901), da demanda do bem substituto a energia elétrica (que foi de 0,0521), e da elasticidade-renda da demanda (que foi de 0,1341), além disso, a Região CentroOeste apresentou a menor elasticidade-preço da tarifa industrial (que foi de 0,0736). J á a Região Sul apresentou a segunda menor elasticidade-preço da tarifa industrial (que foi de - 0,0652) e a segunda menor elasticidade-renda da demanda (que foi de 0,1429), enquanto que a Região Centro-Oeste apresentou a segunda maior elasticidade-preço da demanda de máquinas e equipamentos industriais (que foi de-1,2791).

\section{Considerações Finais}

Este trabalho teve como propósito estimar as elasticidades-preço e renda do consumo de energia elétrica das regiões brasileiras no período de 2000 a 2015, através da utilização de um modelo dinâmico de dados em painel, estimado por meio do método System-GMM, empregando como variáveis, a saber: o consumo, as tarifas de energia elétrica, índices de preço, preço de bens substitutos e a renda das três principais classes de consumo (residencial, comercial e industrial).

Foram avaliadas as elasticidades preço e renda da demanda para os consumidores residencial, comercial eindustrial. Os resultados da estimação das elasticidades preço e renda da demanda para as classes de consumo analisadas mostraram os sinais esperados. Entretanto, os valores das elasticidades variaram bastante entre as regiões e as classes de consumo, contudo, pode-se verificar que a elasticidade-renda da demanda para a classe de consumo industrial foi a que apresentou os maiores valores em todas as regiões analisadas, quando comparado com as outras classes estudadas. Ademais, a classe comercial foi a que registrou os maiores valores da elasticidade-preço da demanda em todas as regiões brasileiras.

Expondo os valores máximos estimados - residencial, comercial e industrial em comparação a outros trabalhos, percebe-se que a elasticidade-preço (salvo o caso comercial da região Nordeste) está dentro dos valores estimado por autores nacionais, como Modiano (1966), Andrade e Lobão (1997) e Schmidt e Lima (1997). Se observados estudos internacionais sobre o tema, os mesmos valores se encaixam com os estudos de Houthakker e J outz (2004), Liu (2004) e Bentzen e Engsted.

Se observados os valores máximos estimados - residencial, comercial eindustrial - em comparação a outros trabalhos sobre o mesmo assunto, observa-se que a elasticidade-renda está dentro dos valores estimados por autores nacionais, como Modiano (1966), Andrade e Lobão (1997) e Schmidt e Lima (1997). Se analisados estudos internacionais sobre o tema, os mesmos valores se aproximam com os estudos de Balestra e Nerlove (1966), Liu (2004) e Bentzen e Engsted (1993).

No caso das regiões examinadas, pode-se aferir que a Região Norte possui, em média, os maiores valores das elasticidades preço e renda da demanda na classe de consumo residencial, o que mostra a sensibilidade nessa classe de consumo, diante de mudanças no preço e na renda desse grupo de estudo. A Região 
Nordeste apresentou os maiores valores das elasticidades preço na classe de consumo comencial e elasticidades preço de máquinas e equipamentos e renda da classe industrial, decorrência da expansão econômica alcançada por essa região nos últimos anos. Já na Região Sudeste, com os resultados apresentados, podeseaferir que os valores das elasticidades preço e renda da demanda para as classes industrial e comercial são os que possuem as menores estimações, o que supõe o uso de outras fontes alternativas de energia, oriundas de termoelétricas, nuclear e fotovoltaica, além da guerra fiscal dos estados, que fez com que empresas migrassem principalmente, para as Regiões Nordeste e Norte. A Região CentroOeste apresentou uma das maiores elasticidades preço da demanda de máquinas e equipamentos industriais e do bem substituto e energia elétrica, consequência do uso de máquinas e equipamentos agrícolas que não possuem muitos concorrentes no mercado e da falta de outras fontes de energia nessa região. Enquanto que a Região Sul está entre os menores valores da elasticidade-renda da demanda estimada para as três classes pesquisadas.

Os valores das elasticidades aqui apresentadas mostram que a região brasileira considerada mais pobre, está mais propensa a mudanças ocorridas no preço e na renda de seus consumidores, enquanto, que no caso contrário, nas regiões consideradas mais desenvolvidas as variações na renda e nos preços são menores. Sendo assim, pode-se avaliar que as elasticidades preço e renda da demanda por energia elétrica das regiões brasileiras nas três classes de consumo, representam as características econômicas dessas regiões, observadas às mudanças no preço e na renda de seus consumidores, o que se pode traçar uma política energética atuante, que beneficie um número maior de consumidores.

Referências

ANDRADE, Thompson A. LOBÃO, Waldir J . A. Elasticidade renda e preço da demanda residencial de energia elétrica no Brasil. Texto para Discussão № 489. Instituto de Pesquisa Econômica Aplicada - IPEA. Rio de Janeiro. J unho de 1997.

AGÊNCIA NACIONAL DE ENERGIA ELÉTRICA (ANEEL). Tarifas médias de consumo residencial de energia elétrica/ Região Período de 2000/2015. Disponível em: <http:// www.aneel.gov.br>. Acesso em: 22 jul. 2017.

Tarifas médias de consumo comercial de energia elétrica/ ${ }^{-}$Região. Período de 2000/2015. Disponível em: <http:// www.aneel.gov.br>. Acesso em: 22 jul. 2017.

Tarifas médias de consumo industrial de energia elétrica/ Região. Período de 2000/2015. Disponível em: <http:// www.aneel.gov.br>. Acesso em: 22 jul. 2017. 
ARELLANO, Manuel; BOND, Stephen. Some Tests of Specification for Panel Data: Mont Carlo Evidence and an Application to Employment Equations. The Review of Economics Studies. Vol. 58. № 2. Pp. 277-297. April, 1991.

BERNDT, Ernst R. The Demand for electricity: Comment and further results. Working Paper № MT-EL 78-021 WP. August 1978.

BERNSTEIN, Mark A. GRIFFIN, J ames. Regional differences in the priceelasticity of demand for energy. National Renewable Energy Laboratory. RAND Infrastructure, Safety and Environment (ISE). Environment, Energy, and Economic Development Program (EEED). 2005.

CAMARGO, Luiz Gustavo Barduco Cugler. O Setor elétrico brasileiro e sua normatização contemporânea. Dissertação (Bacharelado). Centro de Ciências J uńdicas e Sociais Aplicadas. Curso de Direito. Universidade Católica de Santos - UNISANTOS. Santos. 2005.

CASARIN, Ariel A. DELFINO, Maria Eugenia. Price freezes, durables and residential electricity demand. Evidencefrom the Greater Buenos Aires. IAE Business School. Universidad Austral. August 19, 2010.

CHAUDHRY, Azam Amjad. A Panel data analysis of electricity demand in Pakistan. The Labore J ournal of Economics: 15: SE. Pp. 75-106. September 2010.

EMPRESA DE PESQUISA ENERGÉTICA (EPE). Anuário Estatístico de Energia Elétrica. Ministério de Minas e Energia. Rio deJ aneiro. 2016.

Energia. Rio dè J aneiro. 2016.

Balanço Energético Nacional. Ministério de Minas e

FAN, Shun. HYNDMAN, Rob. The Price elasticity of electricity demand in

South Australia. Business and Economic Forecasting Unit. Monash University. Clayton, Victoria. Australia. 2010.

FILIPPINI, Massimo. PACHUARI, Shonali. Elasticities of electricity demand in urban indian households. Centre for Energy Policy and Economics Swiss Federal Institutes of Technology - CEPE. CEPE Working Paper Nr. 16. March 2002.

FUNDAÇÃO GETÚLIO VARGAS (FGV). Índice de Preços por Atacado Oferta Global (IPA-OG) - Combustíveis elubrificantes. Conjuntura Econômica, 2016 a. Disponível em: 〈http:// www.ipeadata.gov.br>. Acesso em: 23 jul. 2017.

. Índice de Preços por Atacado - Oferta Global (IPA-OG) Eletrodoméstico. Conjuntura Econômica, 2016b. Disponível em: <http:// www.ipeadata.gov.br>. Acesso em: 23 jul. 2017. 
. Índice de Preços por Atacado - Oferta Global (IPA-OG) -

Material Elétrico. Conjuntura Econômica, 2016c. Disponível em: <http:// www.ipeadata.gov.br>. Acesso em: 23 jul. 2017.

GOMES, Ludmila de Sá Fonseca e. A demanda por energia elétrica residencial no Brasil: 1999 - 2006. Uma estimativa das elasticidadespreço e renda por meio de painel. Dissertação (mestrado). Faculdade de Economia, Administração e Contabilidade da Universidade de São Paulo de Ribeirão Preto - USP. Ribeirão Preto. 2010.

HOLTEDAHL, Pernille. J OUTZ, Frederick L. Residential electricity demand in Taiwan. Energy Economics 26 (2004) 201-224. Elsevier. J.Eneco. 2003.11.001.

INSTITUTO BRASILEIRO DE GEOGRAFIA E ESTATÍSTICA (IBGE). Índices de PIB e PIB per capita do Brasil. Disponível em: $<h t t p: / /$ www.ibge.gov.br>. Acesso em: 23 jul. 2017.

LABANDERIA, Xavier. LABEAGA, José M. RODRIGUEZ, Miguel. A Residential energy demand system for Spain. Center for Energy and Environmental Policy Research (CEEPR). Massachusetts Institute of Technology - MIT. Reprinted from The Energy J ournal. Vol. 27. № 2. Pp. 87-111. 2006.

MARQUES, Luís David. Modelos com Dados em Painel: revisão de literatura. Centro de Estudos Macroeconômicos e Previsão - CEMPRE. Faculdade de Economia do Porto. Portugal. Outubro, 2000.

MATTOS, Leonando Bornacki de. Demanda de energia elétrica no estado de Minas Gerais: 1970/ 2002. Dissertação (mestrado). Universidade Federal de Viçosa - UFV. Viçosa. Minas Gerais. Brasil. 2004.

MODIANO, Eduardo Marco. Elasticidade-renda e preços da demanda de energia elétrica no Brasil. Texto para Discussão № 68. Departamento de Economia. Maio de 1984.

REISS, Peter C. WHITE, Matthew W. Household electricity demand, revisited. Stanford University. June 14, 2002.

SCHMIDT, Cristiane Alkmin J unqueira. LIMA, Marcos A. M. A Demanda por energia elétrica no Brasil. RBE. Rio de J aneiro. 58(1): 67-99. J an/ Mar 2004.

SIQUEIRA, Marcelo Lettieri. HOLLANDA, Herbetes de. A Demanda setorial por energia elétrica no pós-racionamento de 2001: Previsões de longo prazo para o Nordeste brasileiro. X Encontro Regional de Economia. Maio de 2005. 